\title{
The endoplasmic reticulum-associated degradation is necessary for plant salt tolerance
}

\author{
Lijing Liu ${ }^{1}$, Feng Cui ${ }^{1}$, Qingliang $\mathrm{Li}^{1}$, Bojiao Yin ${ }^{1}$, Huawei Zhang ${ }^{1}$, Baoying Lin ${ }^{1}$, Yaorong Wu ${ }^{1}$, Ran Xia ${ }^{1}$, \\ Sanyuan Tang ${ }^{1}$, Qi Xie ${ }^{1}$

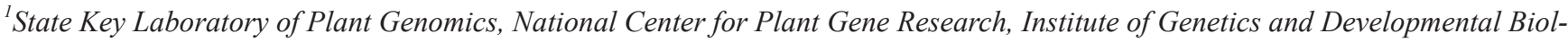 \\ ogy, Chinese Academy of Sciences, No.1 West Beichen Road, Beijing 100101, China
}

Eukaryotic organisms have quality-control mechanisms that allow misfolded or unassembled proteins to be retained in the endoplasmic reticulum (ER) and subsequently degraded by ER-associated degradation (ERAD). The ERAD pathway is well studied in yeast and mammals; however, the biological functions of plant ERAD have not been reported. Through molecular and cellular biological approaches, we found that ERAD is necessary for plants to overcome salt stress. Upon salt treatment ubiquitinated proteins increased in plant cells, especially unfolded proteins that quickly accumulated in the ER and subsequently induced ER stress responses. Defect in HRD3A of the HRD1/ HRD3 complex of the ERAD pathway resulted in alteration of the unfolded protein response (UPR), increased plant sensitivity to salt, and retention of ERAD substrates in plant cells. Furthermore, we demonstrated that $\mathrm{Ca}^{2+}$ release from the ER is involved in the elevation of UPR and reactive oxygen species (ROS) participates the ERAD-related plant salt response pathway.

Keywords: salt stress; ERAD; UPR; $\mathrm{Ca}^{2+}$; ROS

Cell Research (2011) 21:957-969. doi:10.1038/cr.2010.181; published online 28 December 2010

\section{Introduction}

In eukaryotic cells, both luminal and integral membrane proteins entering the secretory pathway are modified and folded in the endoplasmic reticulum (ER) to achieve their native structures. About one-third of newly synthesized proteins are misfolded [1]. Misfolded proteins are retained in the ER until they are either further folded with the help of ER chaperones, or ultimately destroyed by ER-associated degradation (ERAD) [2] The accumulation of unfolded proteins in the ER can be induced by impairment of protein folding derived from mutation, perturbation of protein-protein interactions, or different biotic and abiotic stress stimuli. Accumulated unfolded proteins then cause ER stress [3]. The cell has evolved several mechanisms to recover under ER stress conditions. The misfolded proteins are cleared by the

Correspondence: Qi Xie

Fax: 86-10-64889351

E-mail: qxie@genetics.ac.cn

Received 16 October 2010; revised 26 October 2010; accepted 2 November 2010; published online 28 December 2010
ERAD system. Meanwhile, protein translation is halted to decrease ER burden and a mechanism termed the unfolded protein response (UPR) is activated to increase the expression of ER chaperones and ERAD components to help protein folding and to stimulate degradation of unfolded proteins. If all these processes still cannot restore cell homeostasis within a certain time period, programmed cell death (apoptosis) would be initiated [4]. ERAD is a specific ubiquitin/proteasome system associated with the ER. Similar to the general ubiquitination/degradation systems, it requires ubiquitin-activating enzyme (E1), ubiquitin-conjugating enzyme (E2), ubiquitin ligase (E3), and 26S proteasome, as well as other associated proteins [5]. Because the process occurs in the ER, there are specific complexes involved, including at least some specific E2s and E3 and other associated proteins [6]. It is known that there are two different ERAD complexes in yeast, the HRD1/HRD3 complex and the DOA10 complex. The key components of the HRD1/HRD3 complex are E2s, including UBC1 and UBC7, RING finger type E3 ligase HRD1, and HRD3 that recruits the unfolded proteins for degradation. The key components of the DOA10 complex are E2s, includ- 
ing UBC6 and UBC7, and RING finger type E3 ligase DOA10 [7]. Deletion of these genes would affect the ability of the organism to scavenge unfolded proteins $[8$, 9].

To date, most of the knowledge about ERAD is obtained from yeast and mammals. It has been shown to be involved in a number of human diseases [10-12] and in yeast, ERAD has been shown to be related with various stresses. The defect of UBC7 and UBC1 or the high expression of HRD1 affects yeast responses to $\mathrm{Cd}^{2+}$, a toxic ion to organisms [13]; doal0 and $u b c 7$ mutants are more sensitive to cold than wild type (WT), perhaps through effects on sterol biosynthesis [9]; and overexpression of UBC1, UBC6, and UBC7 increases yeast tolerance to several stresses, including heat, $\mathrm{H}_{2} \mathrm{O}_{2}$, and canavanine [14].

Plants are sessile organisms that must cope with multiple environmental stresses throughout their lives, such as dramatic temperature changes, drought, high salinity, and pathogenic infections $[15,16]$. Among them, salt stress is a serious and global abiotic factor, since high $\mathrm{Na}^{+}$ions in soil are toxic to plants. Under salt stress the activities of many essential enzymes are inhibited, reactive oxygen species (ROS) accumulate, cell division and expansion are blocked, cellular membrane is disorganized, and the osmotic balance is destroyed, all of which finally lead to growth inhibition [17]. Many genes have been identified to be involved in the plant response to salt stress. An excellent example is the set of SOS genes described by Zhu and coworkers [32]. Salt stress induces the accumulation of $\mathrm{Ca}^{2+}$ in the cytoplasm, which is sensed by calcium sensors such as SOS3, which then interacts with SOS2, a protein kinase. The SOS3-SOS2 complex phosphorylates SOS1, a plasma membrane $\mathrm{Na}^{+}$/ $\mathrm{H}^{+}$anti-porter, which effuses excess $\mathrm{Na}^{+}$ions out of cell to reduce the salt damage to plant cells. Previous studies have shown that UPR is induced upon salt stress [18] and that many stress-related genes are upregulated by tunicamycin (TM) and dithiothreitol (DTT), two ER stress inducers $[19,20]$. Some proteins that have functions in both ER quality control (ERQC) and stress responses also have been identified [21-23]. These studies suggest that ER stress and UPR are involved in plant responses to environmental stresses. While studies with a number of substrate proteins have demonstrated that the ERAD pathway exists in plants [24-27], the relationship between ERAD and environmental stress responses has not been explored in plant.

In this study, we identified an ERAD component, HRD3A, in Arabidopsis, which is the homolog of yeast HRD3. By genetic, molecular, and cell biology approaches, we discovered that HRD3A is an active com- ponent of plant ERAD and is involved in plant UPR. By mutational analysis, we found that the intact plant ERAD pathway was necessary for the plants' tolerance to salt stress. Defects of ERAD affected the plant response to salt, and ROS was found to be involved in this process. Furthermore, we found that UPR induced by accumulation of unfolded proteins upon salt and TM treatment was affected by $\mathrm{Ca}^{2+}$ release from the ER.

\section{Results}

\section{Salt treatment quickly induces the ER stress response}

It is known that in mammals and yeast, the ubiquitin/26S proteasome system (UPS) and especially ERAD are involved in degrading the misfolded proteins induced by different physical and biological invasions [3]. In plants, the UPS has been increasingly implicated in plant responses to environmental stresses, and there is some indirect evidence indicating that ERAD indeed occurs in plants $[24,26]$, but whether the ERAD pathway is involved in plant tolerance to abiotic stresses is unclear. To gain further insight into the relationship between ERAD and abiotic stresses in plants, 2-week-old WT seedlings were treated with salt, and protein extracts were prepared for western blot analysis. The level of total ubiquitinated proteins was upregulated by salt, especially at 3 and 6 $\mathrm{h}$ after treatment (Figure 1A), indicating that a number of proteins were quickly ubiquitinated and perhaps degraded through UPS. We then analyzed the expression of ER stress marker genes that reflects the accumulation of unfolded proteins in the ER. The expression levels of $B I P 1 / 2$ as well as $C N X 1$ are largely increased by $\mathrm{NaCl}$ treatment (Figure 1B). We conclude that, salt treatment altered the levels of total ubiquitinated proteins and promoted the UPR in the plant. These results suggest that plant ERAD may function in salt stress responses.

\section{HRD3A is the only functional homolog of yeast HRD3 in Arabidopsis}

To define the relationship between ERAD and the plant salt response, genes encoding protein homologs to yeast ERAD components were identified by Blast analysis. Candidate genes (Supplementary information, Figure S1A) were further analyzed by reverse genetics to determine whether they participate in the plant ERAD and salt stress response. In this study, we explored in detail the function of the plant ERAD component HRD3. Through protein alignments with yeast HRD3, two putative HRD3 homologs, named HRD3A (at 1 18260) and HRD3B (at1g73570), were identified in Arabidopsis (Supplementary information, Figure S1D). The T-DNA insertion mutant lines of these two genes were obtained from the 

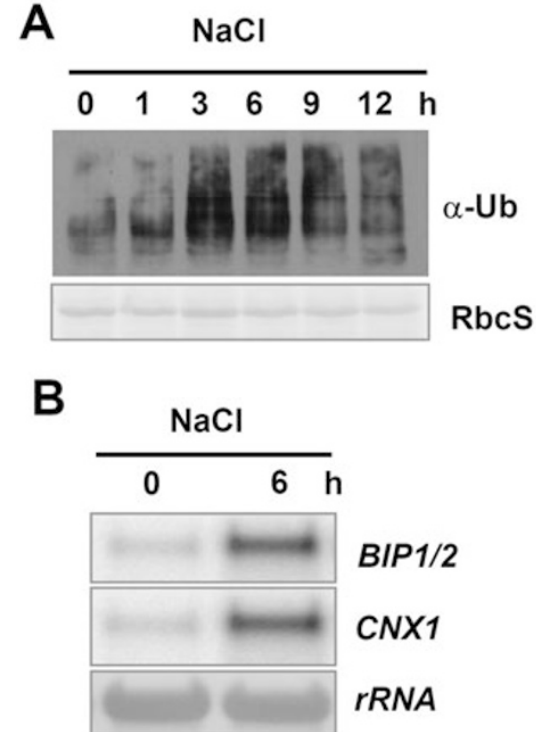

Figure 1 Salt-induced UPR in plants. The 2-week-old seedlings were treated in $\mathrm{MS}$ liquid medium containing $300 \mathrm{mM} \mathrm{NaCl}$, and samples were collected at different time points. Equal amounts of total proteins were separated by SDS-PAGE and analyzed by immunoblots. Ponceau $\mathrm{S}$ staining of RbcS served as a loading control. For RNA analysis, $15 \mu \mathrm{g}$ of total RNA from each sample were applied for Northern blotting. (A) Western blot analysis by using an ubiquitin-specific antibody. (B) Expression of ER stress chaperone genes were detected with ${ }^{32} \mathrm{P}$-labeled BIP1/2 and CNX1 probes. The bottom panel shows methylene blue-stained $28 \mathrm{~S}$ rRNA as a loading control.

Arabidopsis Biological Resource Center (ABRC), stock center. Homozygous mutant lines of the two genes were isolated and identified as hrd3a-1 (SALK_109430) and hrd3b-1 (SALK_054396) (Figure 2A and Supplementary information, Figure S2A). Loss of specific RNA expression in the mutants was confirmed by RT-PCR analysis (Supplementary information, Figure S1B and S1C). To examine whether both HRD3A and HRD3B are active components of the ERAD complex in Arabidopsis, $h r d 3 a-1$, and $h r d 3 b-1$ mutants were grown on medium containing $0.2 \mu \mathrm{g} / \mathrm{ml} \mathrm{TM}$, an ER stress inducer through inhibiting protein glycosylation [28]. Surprisingly, only the growth of $h r d 3 a-1$, but not $h r d 3 b-1$, was sensitive to TM (Supplementary information, Figure S2B). To determine the reason for the insensitivity of $h r d 3 b-1$ to $\mathrm{TM}$, the $H R D 3 B$ cDNA was cloned from WT young seedlings and sequenced, but the result was not consistent with the CDS predicted by TAIR (http://www.arabidopsis.org). In fact, the second intron was retained in the cDNA, causing the protein to be terminated prematurely (Supplementary information, Figures S1D and S2A). To investigate whether the $H R D 3 B$ transcript is alternatively spliced, RT-PCR analysis was performed with primers designed nearby or in the second intron (Supplementary information, Figure S2A), by using RNA isolated under different treatments and RNA from different tissues, including cauline leaves, rosette leaves, stems, roots, siliques, and flowers. We found that the second intron of $H R D 3 B$ was present in transcripts from all tissues and transcripts under different treatments (Supplementary information, Figure S2C and S2D). Combining the genetic and molecular data, we conclude that $H R D 3 B$ may be a pseudogene or perhaps encodes only a truncated protein, and $H R D 3 A$ is likely the only known functional homolog of yeast HRD3 in Arabidopsis.

\section{HRD3A is involved in plant tolerance to ER stress and $U P R$}

Since there is only one functional HRD3 in Arabidopsis, HRD3A, we then focused on exploring its function. To ensure that the phenotype of $h r d 3 a-1$ is indeed the result of a null mutation of $H R D 3 A$, we identified the other allele, $h r d 3 a-2$ (SALK_050640). The expression of the HRD3A gene was also abolished in this line (Figure 2A and Supplementary information, Figure S1B). On Murashige and Skoog (MS) standard growth medium, no obvious differences were observed between the WT and both $h r d 3 a$ mutants. However, on plates containing different concentrations of TM, both $h r d 3 a-1$ and $h r d 3 a-2$ showed more sensitivity than the WT in a dosage-dependent manner (Figure 2B and 2C). On $0.15 \mu \mathrm{g} / \mathrm{ml} \mathrm{TM}$ plates, more than $60 \%$ of the WT seedlings grew well. By contrast, the percentages in mutants were less than $25 \%$. On $0.25 \mu \mathrm{g} / \mathrm{ml} \mathrm{TM}$ plates, the growth of almost all seedlings of both mutants was blocked, while about $25 \%$ of the WT seedlings still had green true leaves. This result indicates that the $h r d 3 a$ mutants are sensitive to TM. Since the $h r d 3 a-1$ and $h r d 3 a-2$ mutants had similar phenotypes under all stresses tested (Figures 2B, 4B, 6B and Supplementary information, Figure S5), we simply chose $h r d 3 a-1$ for subsequent in-depth molecular analyses.

The ER stress would trigger UPR [5, 29]. A sign of induced UPR is the high expression of ER chaperones genes, such as BIP1/2, CNX, and PDIL1-1. The 2-weekold seedlings of the WT and $h r d 3 a-1$ mutant were treated with $5 \mu \mathrm{g} / \mathrm{ml} \mathrm{TM}$, and total RNA was extracted for northern analysis. ER chaperone gene expression was induced in the WT, which is consistent with a previous study [18], and in $h r d 3 a-1$ the induced level was much higher than in the WT with prolonged increases for all the three marker genes analyzed (Figure 2D). Therefore, we propose that the defect in the putative ERAD component HRD3A in plants enhances UPR under ER stress. 

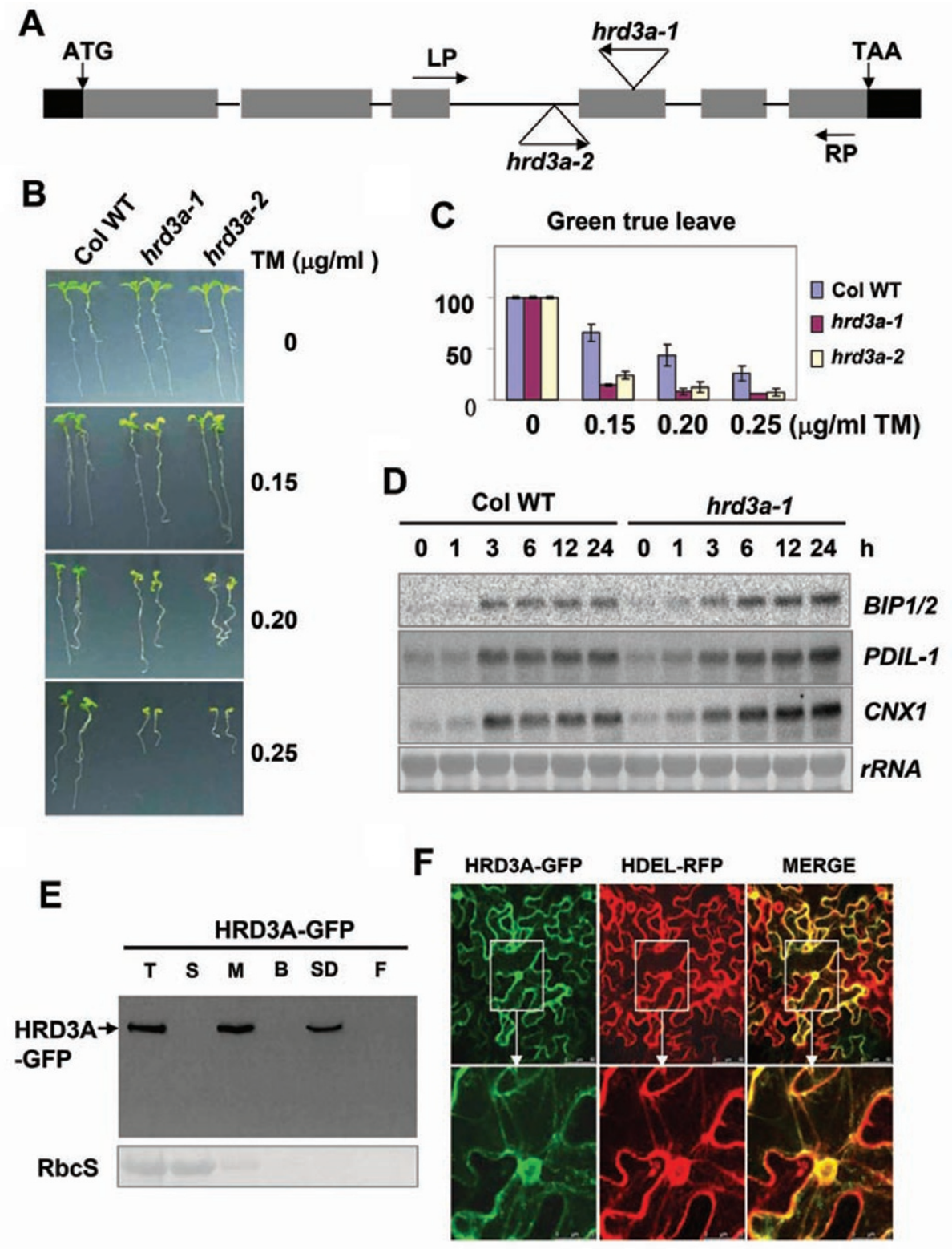

Figure 2 The mutant plant of ER membrane protein HRD3A is sensitive to ER stress inducer, tunicamycin. (A) Schematic diagram of the HRD3A gene structure and primers for RT-PCR. Closed boxes represented exons; lines between closed boxes represent introns; and black boxes represent the 5'- and 3'-UTR. The two null mutants, hrd3a-1 and hrd3a-2, were identified. $\mathrm{LP}$, forward primer; RP, reverse primer. (B) Growth of WT and mutant plants on MS medium containing a range of concentrations of $\operatorname{TM}(0,0.15,0.20$, and $0.25 \mu \mathrm{g} / \mathrm{ml})$. Seeds were germinated and grown for 8 days on plates with or without TM, and representative plants are shown. Percentages are means $(n=60$ each) of three independent repeats. (C) Quantitative analysis of $h r d 3 a$ mutants and WT seedlings with green true leaves on plates containing a range of concentrations $(0,0.15,0.2$, and $0.25 \mu \mathrm{g} / \mathrm{ml}$ ) of TM. (D) Expression patterns of transcripts of ER chaperones in response to TM treatment. The 2-weekold seedlings were treated with MS media containing $5 \mu \mathrm{g} / \mathrm{ml} \mathrm{TM}$ for different times. Total RNA (15 $\mu \mathrm{g})$ from each sample was hybridized with ${ }^{32} \mathrm{P}$-labeled BIP1/2, PDIL-1, and CNX1 probes. The bottom panel shows methylene blue-stained $28 \mathrm{~S}$ rRNA as a loading control. (E) Cell fractionation assays of HRD3A. 35S:HRD3A-GFP was infiltrated into tobacco leaves and the samples were collected after $3 \mathrm{~d}$. HRD3A-GFP was detected using an anti-GFP antibody (top panel). Ponceau S staining of the RbcS is shown as a loading control (bottom panel). B, buffer-extracted fraction; F, final membrane fraction; M, membrane fraction; S, soluble fraction; SD, SDS-extracted fraction; T, total extract. (F) Co-localization of HRD3A-GFP with HDEL-RFP. Tobacco leaves were co-infiltrated with 35S: HRD3A-GFP and 35S: HDEL-RFP. Signals were observed directly under a confocal microscope after $3 \mathrm{~d}$. The photographs in the bottom panel are the enlargements of the nuclear area of corresponding frames as indicated by the arrows. 


\section{HRD $3 A$ is an ER-localized protein}

HRD3A is the homolog of yeast HRD3, an ER-localized membrane protein. By using the SMART program, we could not identify a typical membrane localization sequence in HRD3A. Therefore, cell fractionation and HRD3A-GFP fusion analysis were performed to detect HRD3A localization. First, we constructed an HRD3AGFP fusion cassette under the control of a cauliflower mosaic virus $35 \mathrm{~S}$ promoter. This construct was transiently expressed in Nicotiana benthamiana leaf cells by agrobacteria infiltration [30]. Cell fractionation and protein gel blot assays showed that HRD3A-GFP was present in the microsomal fraction, but not in the soluble fraction (Figure 2E). The association of HRD3A-GFP with the membranes was further confirmed by resuspension of the membrane fraction in detergent-free and detergent-containing buffers for extensive cell fractionation analysis. HRD3A-GFP was only removed from the microsomal fractions by detergent treatment (Figure 2E). Therefore, we conclude that HRD3A is associated with the intracellular membranes.

The subcellular localization of HRD3A was further examined by microscopy. After 3 days of incubation, expression of the HRD $3 A-G F P$ gene was examined under a confocal laser scanning microscope. As shown in Figure 2F and Supplementary information, Figure S3, HRD3A-GFP exhibited typical ER localization. The ER localization of HRD3A-GFP was further confirmed by co-expression with the ER marker, HDEL-RFP. Colocalization of HRD3A and the ER marker was observed, as indicated by the perfect overlay of the RFP and GFP fluorescent signals (Figure 2F). Both the biochemical and cellular analyses indicate HRD3A is an ER-localized protein.

\section{HRD $3 A$ is an active component of ERAD in plants}

Recent studies revealed that two mutated BR receptors, bri1-5 and bri1-9, are ERAD substrates in Arabidopsis $[25,26]$. To further explore the function of HRD3A in ERAD, we examined the effect of the $h r d 3 a$ mutation on bri1-5 and bri1-9. The hrd3a-1 bril-5 double mutants have larger rosette leaves with noticeable petioles than that of the bri1-5 single mutant (Figure 3A). Meanwhile, the $h r d 3 a-1$ bril-9 double mutant also suppressed the bril-9 small rosette phenotype (Figure 3B). Consistent with the morphological phenotypes, the two double mutants regained partial sensitivity to epi-brassinolide (eBL), an active form of BR (Figure 3C and 3D), as measured at the biochemical level by BL-induced BES1 dephosphorylation assays [31].

It had been shown that accumulated bri1-5 and bri1-9 proteins in the ER may saturate the ERQC machinery, and those proteins would leak out of the ER to the cell surface where they can receive BR signals $[25,26]$. Therefore, the protein levels of bri1-5 and bri1-9 were examined in both double mutants. Both bri1-5 and bri1-9 proteins were accumulated at higher levels in double mutants compared with that in their single mutants, WT and in the hrd 3a-1 mutant (Figure 3E).

In addition, we tested the effect of HRD3A on another known ERAD substrate MLO-1, which is a mutant form of Barley powdery mildew resistance o (MLO) [24]. 35S:Myc-MLO-1 and 35S:GFP plasmids were co-transfected into Arabidopsis mesophyll protoplasts prepared from WT or $h r d 3 a-1$ lines. Total proteins were extracted from the protoplasts 1 day after transfection and analyzed by SDS-PAGE. As shown in Supplementary information, Figure S4, the level of Myc-MLO-1 proteins was increased dramatically in the $h r d 3 a-1$ mutant compared with that in the WT, whereas the level of the Myc-MLO-1 mRNA was not different between the two samples. Collectively, our results indicate that HRD3A is an active component of ERAD in Arabidopsis.

\section{ERAD defects increase the plant sensitivity to salt}

Our results thus far indicate that HRD3A is an important component of the Arabidopsis ERAD complex. Defects in ERAD are related to different stress responses in yeast $[12,14]$ and important diseases in mammals $[11,12]$. Our current observations of salt treatment in Arabidopsis indicated that ERAD was involved in plant salt stress response. As HRD3A is an active component of the ERAD complex, it is expected that the $h r d 3 a$ mutant plants would have altered responses to high-salt conditions. Therefore, seedlings grown on $125 \mathrm{mM} \mathrm{NaCl}$ plates for 2 weeks were examined. Seedlings of both $h r$ $d 3 a$ mutants were smaller and weaker than the WT seedlings (Figure 4A). The green seedlings of the WT were about $55 \%$ while less than $25 \%$ of the seedlings of both mutants were green (Figure 4B). Similar results were observed when the plants were grown on medium with 125 $\mathrm{mM} \mathrm{KCl}$, while no obvious differences were detected when $250 \mathrm{mM}$ mannitol (equal to the same osmotic effect of $125 \mathrm{mM} \mathrm{NaCl}$ or $\mathrm{KCl}$ ) was added to the medium (Supplementary information, Figure S5). The results above suggest that the HRD3-dependent stress response is salt ion specific rather than a result of osmotic stress.

It has been shown that ubiquitinated proteins increase upon salt treatment in this work. We then examined the status of ubiquitinated proteins in the $h r d 3 a-1$ mutant with or without salt treatment. The total level of ubiquitinated proteins was already higher in the $h r d 3 a-1$ mutant than the WT without salt treatment. While the ubiquitinated proteins accumulated in both lines upon salt treat- 

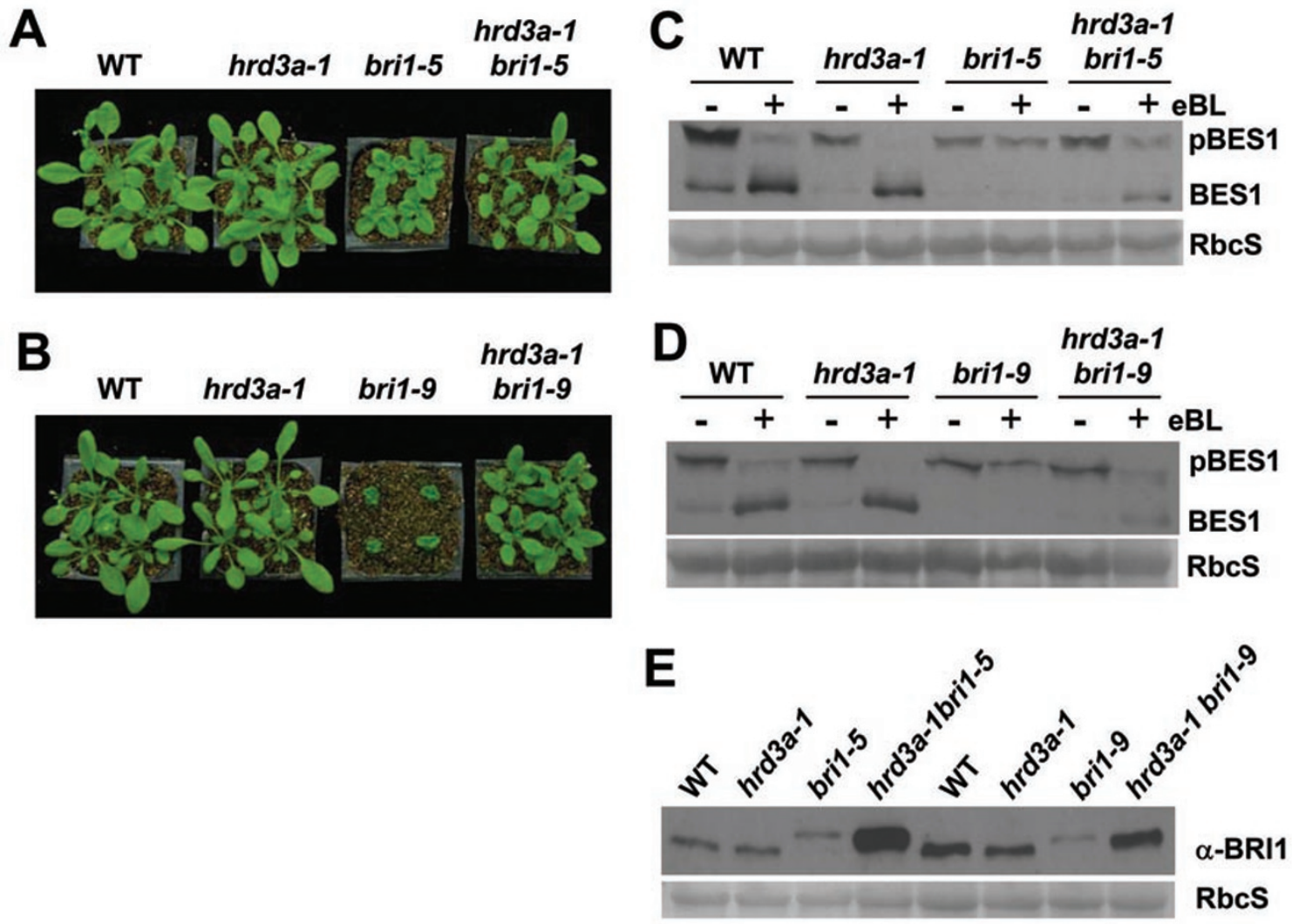

Figure 3 The hrd3a-1 mutant suppressed the bri1-5 and bri1-9 phenotypes by affecting their protein accumulation. (A) The 4-week-old soil-grown plants of sibling WT, hrd3a-1, bri1-5, and hrd3a-1 bri1-5 double mutant. (B) The 4-week-old soil-grown plants of sibling WT, hrd3a-1, bri1-9, and hrd3a-1 bri1-9 double mutant. (C, D) Immunoblot analysis of the BES1 phosphorylation status in seedlings treated with or without $10 \mu \mathrm{M}$ epi-brassinolide (eBL). Total proteins were extracted from the 2-weekold seedlings treated with or without $10 \mu \mathrm{M}$ eBL for $1 \mathrm{~h}$ in liquid MS, separated by $10 \%$ SDS-PAGE, and analyzed by immunoblotting with anti-BES1 antibody. pBES1 is the phosphorylated form of BES1. Coomassie blue staining of RbcS served as the loading control. (E) Immunoblot analysis of the abundance of BRI1 and its mutant forms in plants are shown in (A) and (B). Equal amounts of total proteins extracted were separated by SDS-PAGE and analyzed by immunoblots using an anti-BRI1 antibody. Coomassie blue staining of RbcS served as a loading control.

ment, the level was higher in the hrd3a-1 mutant than in the WT (Figure 4C). This result suggests that there may be more unfolded proteins in $h r d 3 a$ mutants than in WT plants upon salt treatment. High accumulation of unfolded proteins in the cell would induce UPR. Thus, total RNA was extracted from salt-treated seedlings and blots were probed with ER chaperone genes. The expression of ER chaperone genes was induced in both $h r d 3 a-1$ and WT, while the induction levels of all three chaperones in $h r d 3 a-1$ was much higher and lasted for a longer period of time than in the WT (Figure 4D). The fact that more unfolded proteins accumulated in the $h r d 3 a$ mutant may explain the sensitive phenotype of $h r d 3 a$ mutants to salt.

$\mathrm{Ca}^{2+}$ release from the ER is involved in the elevation of $U P R$

Next, we sought to explore the signaling pathway related to the function of HRD3A in the salt response. Previous studies have shown that $\mathrm{Ca}^{2+}$, as a second messenger, is released from the ER under salt treatment to induce salt responses in plant cells $[32,33]$. In our study, we found the expression of ER chaperone genes was highly induced in the $h r d 3 a$ mutant upon salt treatment (Figure 4E). To address whether the high-level induction of the UPR was also affected by $\mathrm{Ca}^{2+}$ release from ER, we conducted the $\mathrm{Ca}^{2+}$ release inhibition experiment. Two-week-old seedlings of both the WT and hrd $3 a-1$ mutant were treated with salt with or without $100 \mu \mathrm{M}$ 2-aminoethoxydiphenyl borate (2-APB), an inhibitor of $\mathrm{InsP}_{3}$-induced calcium release [34]. The RNA gel blot analysis showed that 2-APB blocked the induction of the $B I P 1 / 2$ gene upon salt treatment in both WT and $h r d 3 a-1$ samples. The expression of the $B I P 1 / 2$ gene upon treatment with both $\mathrm{NaCl}$ and 2-APB was reduced to almost 
the same level between the WT and hrd $3 a-1$ samples (Figure 5A). This indicates that $\mathrm{NaCl}$ indeed induced UPR through the release of $\mathrm{Ca}^{2+}$ from the ER. The expression of $B I P 1 / 2$ was blocked by the inhibitor of $\mathrm{Ca}^{2+}$ release for two possible reasons. Either unfolded proteins had accumulated in the ER upon salt treatment due to $\mathrm{Ca}^{2+}$ release from the ER, as previous studies had shown that reduced $\mathrm{Ca}^{2+}$ in the ER induces ER stress, or the ER stress signal could not be transmitted to the nucleus to stimulate the UPR when the release of $\mathrm{Ca}^{2+}$ from the ER is inhibited. To test these hypotheses, we treated seedlings with TM, a direct ER stress inducer by inhibiting protein glycosylation and inducing accumulation of unfolded proteins. Similar to salt treatment, 2-APB also blocked the induction of the BIP1/2 gene in both the WT and $h r d 3 a-1$ samples (Figure 5B). Thus, the induction of
UPR under salt-induced ER stress requires $\mathrm{Ca}^{2+}$ release from the ER.

ROS is involved in the ERAD-related plant salt response pathway

When a plant faces a high-salt environment, its growth is arrested and prolonged salt treatment also leads to plant death. It is known that salt stress generates ROS in plants $[35,36]$, and over-production of ROS in animals results in oxidative stress and accumulation of unfolded proteins $[37,38]$. To determine whether the production of ROS in ERAD deficient plant is different from that of WT plants upon salt treatment, the $\mathrm{H}_{2} \mathrm{O}_{2}$ accumulation in the hrd3a-1 mutant upon salt treatment was visualized by 3,3-diaminobenzidine (DAB), a chemical which converts to dark-brown polymers in the presence of $\mathrm{H}_{2} \mathrm{O}_{2}$
A

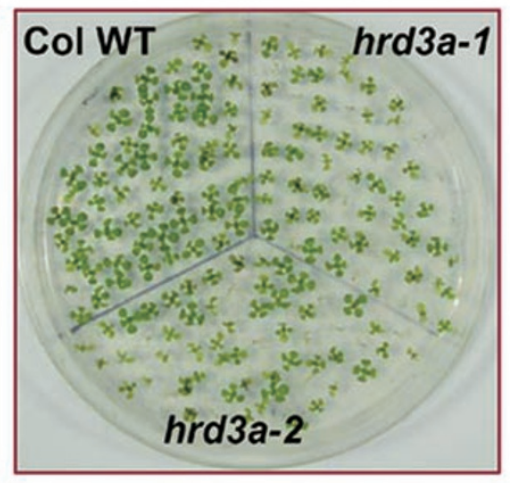

C

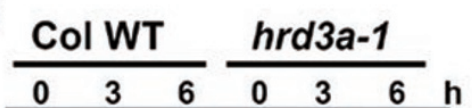

B

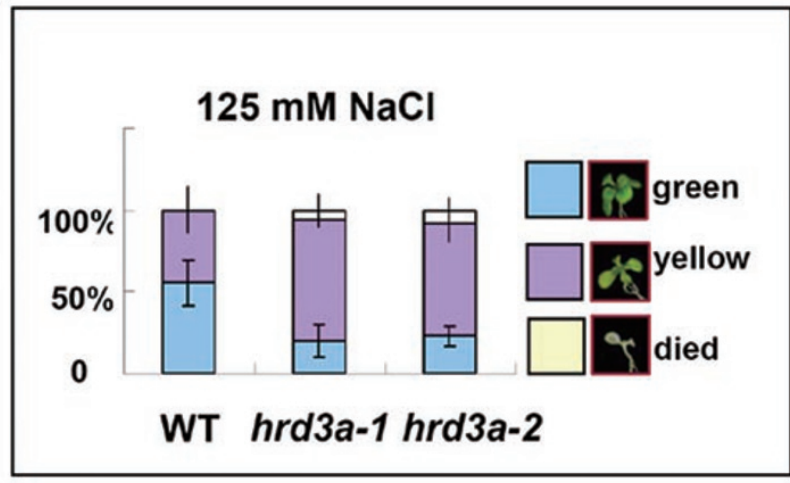

D

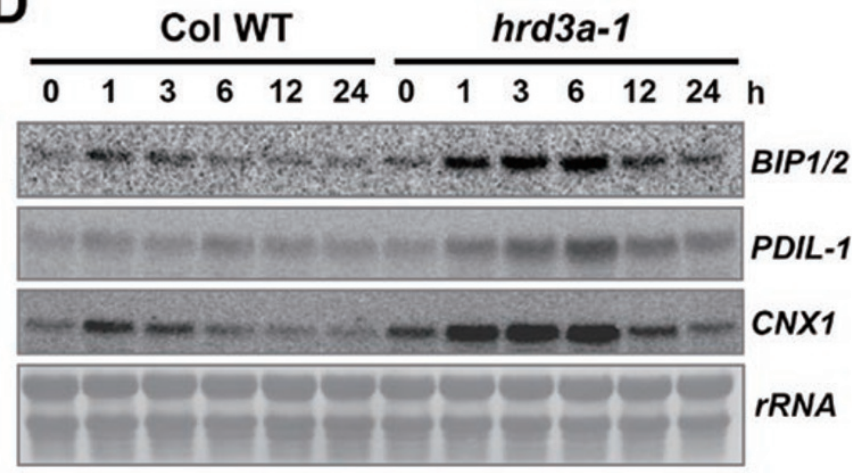

Figure 4 The inhibition of ERAD seriously affected plant responses to $\mathrm{NaCl}$. (A) Growth of different lines of plants on $\mathrm{MS}$ medium plates containing $125 \mathrm{mM} \mathrm{NaCl}$. Seeds were germinated and grown for 2 weeks. (B) Quantitative analysis of different plant phenotypes of $h r d 3 a$ mutants and WT as shown in (A); percentages are means ( $n=60$ each) of three independent repeats. (C) Western blot analysis of hrd3a-1 mutant and WT treated with $300 \mathrm{mM} \mathrm{NaCl}$ for different time points using antiubiquitin antibody. Plant was grown for 2 weeks, and then treated in MS liquid medium containing $300 \mathrm{mM} \mathrm{NaCl}$. Proteins were extracted and immunoblotted with anti-ubiquitin antibody. (D) Expression patterns of transcripts of ER chaperones in response to $\mathrm{NaCl}$ treatment. Plants were grown for 2 weeks, and then treated with medium containing $300 \mathrm{mM} \mathrm{NaCl}$ for different time points. Total RNA $(15 \mu \mathrm{g})$ from each sample was hybridized with ${ }^{32} \mathrm{P}$-labeled BIP1/2, PDIL-1, and CNX1 probes. The bottom panel shows methylene blue-stained $28 \mathrm{~S}$ rRNA as a loading control. 


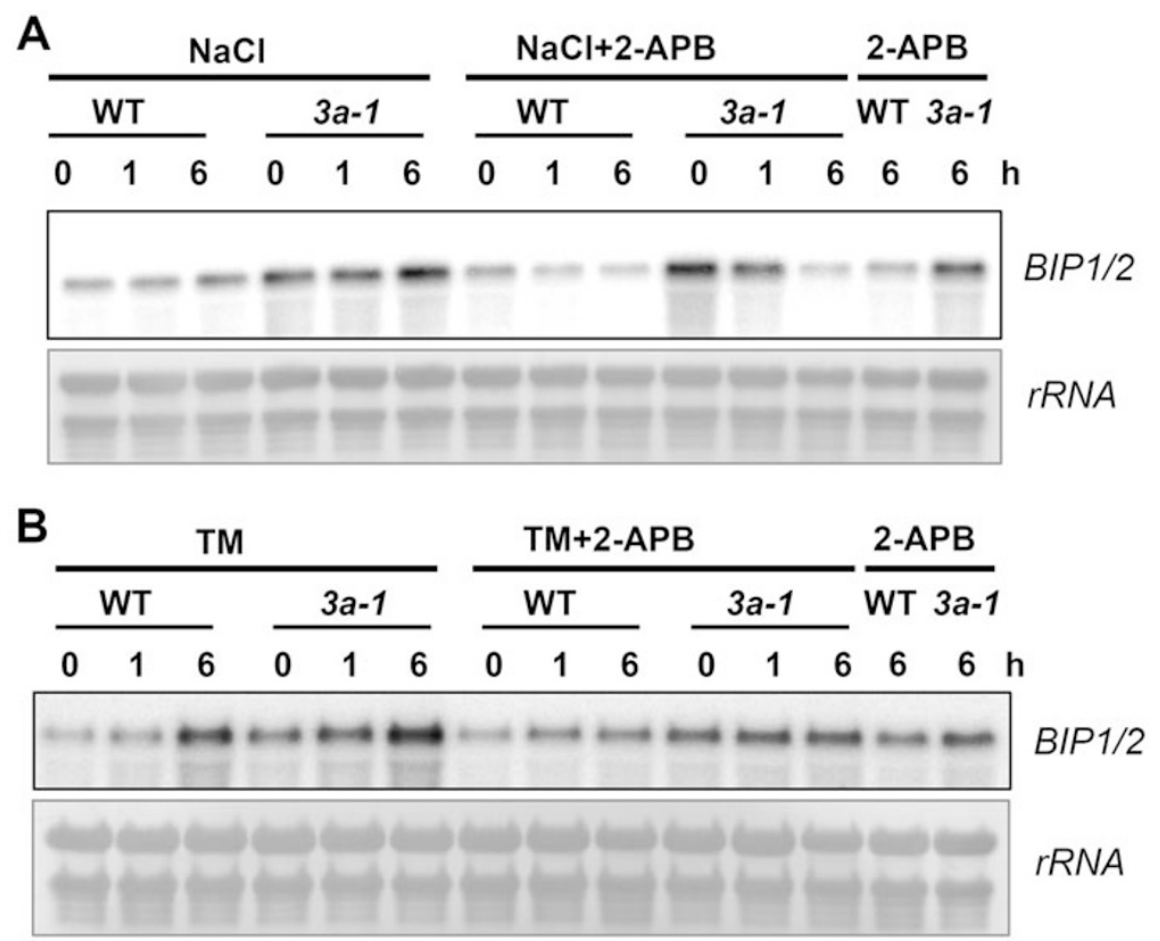

Figure $5 \mathrm{Ca}^{2+}$ release from the ER is a signal to initiate UPR. Expression patterns of ER chaperone BIP1/2 in response to $\mathrm{NaCl}$ and TM treatments. The 2-week-old plants were subjected to different treatments and samples were collected at different time points. Total RNA $(15 \mu \mathrm{g})$ from each sample was hybridized with ${ }^{32} \mathrm{P}$-labeled BIP1/2 probe. The bottom panels show methylene blue-stained 28S rRNA as a loading control. (A) Treatment of $300 \mathrm{mM} \mathrm{NaCl}$ with or without $100 \mu \mathrm{M} 2-\mathrm{APB}$. (B) Treatment of $5 \mu \mathrm{g} / \mathrm{ml}$ TM with or without $100 \mu \mathrm{M} 2-\mathrm{APB}$.

[39]. The $\mathrm{H}_{2} \mathrm{O}_{2}$ level in hrd3a-l was higher than in WT both with and without salt treatment (Figure 6A). The salt treatment promoted $\mathrm{H}_{2} \mathrm{O}_{2}$ accumulation, especially in hrd $3 a$ plants. This result indicates that HRD3A is at least important for removing the source of ROS production in plant cells, and perhaps this is the reason why the hrd $3 a$ plants showed arrested growth and died more quickly under the salt treatment condition. The chemical paraquat, an inducer of oxidative stress, can induce cell death in both plants and animals [40, 41]. To determine whether paraquat can be more harmful to $h r d 3 a$ mutant plants, hrd $3 a-1, h r d 3 a-2$, and WT seeds were plated and grown on MS medium containing $0.2 \mu \mathrm{M}$ paraquat. After being grown for 2 weeks, the $h r d 3 a-1$ and $h r d 3 a-2$ mutants each showed an obvious sensitive phenotype to paraquat, as most of the mutant seedlings were small, and the leaves were bent (Figure 6B). The vast majority of the WT seedlings (98\%) grew well, but less than $10 \%$ of the seedlings of the two mutants were larger plants (Figure 6C). Continued treatment also led to faster death of those hrd $3 a$ mutant plants, indicating that ERAD was necessary for clearing ROS-induced accumulation of unfolded proteins.
Taking these findings altogether, we conclude that salt stress activated ROS signals and induced the accumulation of unfolded proteins in the ER. The removal of those unfolded proteins by the ERAD is important for plant tolerance to salt. When a key component of the ERAD, HRD3A, was defective in the plant, accumulation of unfolded proteins strongly induced UPR that required $\mathrm{Ca}^{2+}$ release from the ER.

\section{Discussion}

To date, knowledge of ERAD has mainly been obtained from studies of yeast and mammalian systems, but little is known about plant ERAD components and their effects on plant development and growth. Genome sequence and several microarray experiments have revealed that ERAD components similar to yeast and mammals are present in plants $[19,20]$. We discovered that HRD3A is the only active HRD3 homolog in Arabidopsis; however, we could not detect the complementation of yeast $h r d 3$ mutant by HRD3A (data not shown), which may be due to the low similarity between these two proteins (29.6\%). HRD3A is an ER-localized protein 

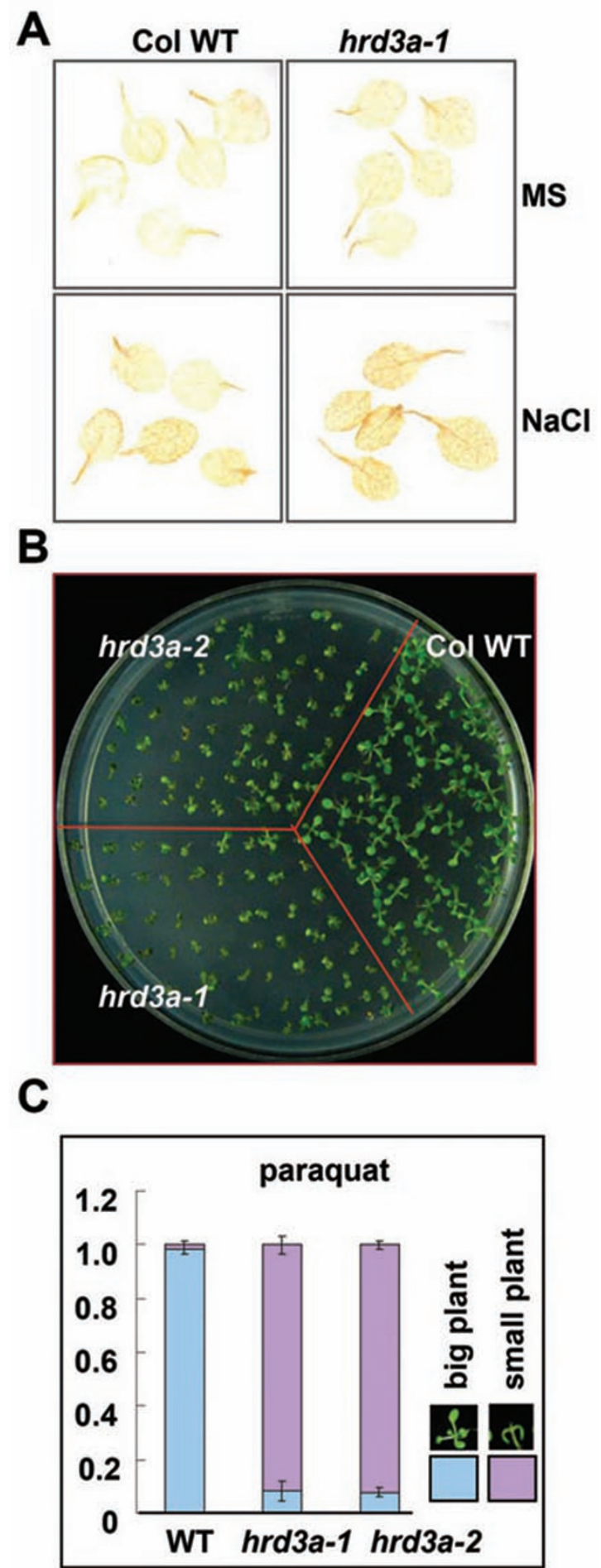

Figure 6 ROS is involved in the ERAD-related plant salt response pathway. (A) 3,3-diaminobenzidine staining of the $\mathrm{H}_{2} \mathrm{O}_{2}$ levels in WT and hrd3a-1 mutant plants with or without $\mathrm{NaCl}$ treatment. For $\mathrm{NaCl}$ treatment, 3-week-old seedlings were treated with MS medium containing $300 \mathrm{mM} \mathrm{NaCl}$ for $6 \mathrm{~h}$. (B) Growth phenotype of WT and hrd3a mutants germinated and grown for 2 weeks on MS medium containing $0.2 \mu \mathrm{M}$ Paraquat. (C) Quantitative analysis of the different phenotypes of (B). Percentages are means ( $n=60$ each) of three independent repeats. and the stability of several ERAD substrates was increased in $h r d 3 a$ mutant. In the case of the BR receptor, however, we could not detect the cytoplasmic form of the BRI1 mutants in two double mutants, hrd 3a-1 bril-5 and hrd3a-1 bri1-9 by Endo $\mathrm{H}$ digestion. This result could be due to the low amounts of the mutant proteins, or the different effect of ERAD component deficiency in comparison to the changes in upstream components affecting glycosylation [25].

Salt stress is one of the most serious environmental stresses to plants. ERAD has been shown to be involved in a number of human diseases and stress responses in yeast. We demonstrated, here, that ERAD is involved in the plant response to salt. In mammals, under ER stress BIP protein is occupied by the accumulated misfolded proteins in ER, and the interaction of BIP and PERK is disturbed. PERK is then activated to inhibit the translation of most proteins [29]. The biological effect is the cell-cycle arrest. If this pathway is conserved in plant, it might be able to explain the smaller phenotype of $h r d 3 a$ under salt stress. Our results showed that the total ubiquitinated proteins accumulated to a higher level in $h r d 3 a$ than in WT upon salt treatment, this may be because of the fact that proteins ubiquitinated by ERAD only contribute to part of total ubiquitinated proteins or the result of the compensation by other ERAD complex, such as uncharacterized DOA10 complex in plant. The other explanation is that HRD3A mutation may not affect the ubiquitination of misfolded proteins, but only their degradation by ERAD.

Signal transduction under ER stress to induce UPR has been well studied in yeast and mammalian cells. In yeast, UPR is activated through the IRE1-HAC1 pathway [2], and compared with yeast, the induction of UPR in the mammalian system is a more complicated event. There are at last three ER stress sensors, activating transcription factor 6, IRE1a, or IRE1b, and PERK that activate UPR through distinct ways [29]. The bZIP28 and bZIP60 proteins have also been shown to function as ER stress sensors in plants [21, 42-44]. Whether plants utilize all those sensor systems will need to be addressed in the future. Here, we identified $\mathrm{Ca}^{2+}$, an important second messenger in cells, as essential for the induction of UPR upon salt treatment in plants, especially when ERAD is deficient. However, whether the induction of UPR through $\mathrm{Ca}^{2+}$ signals and bZIP28 or bZIP60 signals are related to each other or belong to completely different pathways remains to be determined. Indeed, $\mathrm{Ca}^{2+}$ signals are known to be involved in other types of biotic and abiotic stresses, such as drought, cold, heat, immersion, and several different infectious diseases. Although UPRrelated diseases in plants have been documented [45], 
whether ERAD is involved in all those stress signals are not yet known.

ROS are generated immediately when plants are under environmental stress. They can both function as a signal to activate stress-related responses and cause oxidative damage to cells [46]. High salinity in soil is a serious environmental stress to plants and induction of ROS accumulation in plants has also been observed [35, 47]. However, how ROS accumulation causes plant growth inhibition and cell death under salt stress has not been well defined. Our results provide evidence that the damage to plants may be partially through ROS-induced accumulation of misfolded proteins in cells. It is known that oxidative stress enhances ROS production through the oxidation and reduction of disulfide bonds [48]. It is well known that the formation of disulfide bonds is important for correct protein folding. Protein folding in the ER is a dynamic process, and proteins require reducing capacity to allow refolding when disulfide bonds form incorrectly. The accumulation of ROS is also known to occupy most NADPH in cells, resulting in the accumulation of misfolded proteins. When these misfolded proteins are not dealt with properly, growth inhibition and cell death occur.

All current evidence indicates that an ERAD mechanism is important for plants to survive under high-salt conditions. According to our study and previous work, we propose the following model in plants (Figure 7). The misfolded proteins accumulate in the ER upon salt

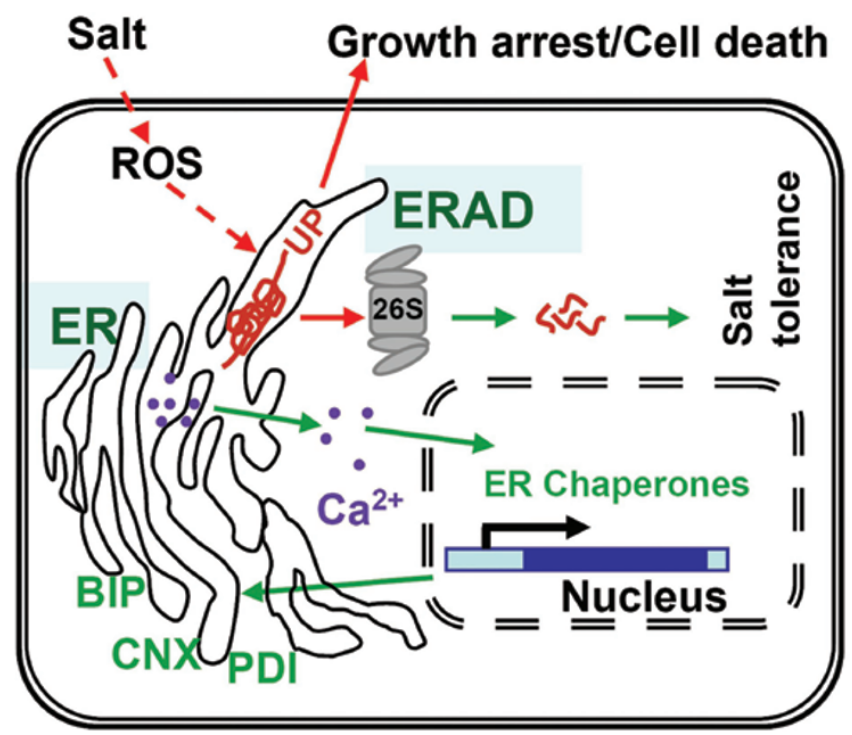

Figure 7 Proposed model for the role of ERAD in the salt signaling pathway in plants. UP: unfolded protein. treatment, which may be mediated through ROS signaling pathways. Those misfolded proteins are recognized by ERAD and finally degraded by the $26 \mathrm{~S}$ proteasome. Plants can also alleviate salt stress by inducing the expression of ER chaperones to help the misfolded proteins to refold. If ERAD is deficient under salt stress, the plant response to salt is changed. A much stronger UPR would arise to induce additional ER chaperones, and this process requires the release of $\mathrm{Ca}^{2+}$ from the ER. Consequently, plant growth is inhibited, which may be the result of inhibition of translation of essential proteins in the cells, and prolonged salt treatment would also cause plant cell death by activating apoptosis-like programmed cell death.

\section{Materials and Methods}

\section{Plant materials and growth conditions}

The Arabidopsis thaliana Columbia ecotype was used for this study. Seeds were surface sterilized with $10 \%$ bleach for 15 min and washed five times with sterile water. Sterile seeds were suspended in $0.2 \%$ agarose and plated on MS medium (the MS medium used in this study is actually $1 / 2 \mathrm{MS}$ ). These plates were stratified in darkness for 3 days at $4{ }^{\circ} \mathrm{C}$, after that transferred to a tissue culture room at $22{ }^{\circ} \mathrm{C}$ under a 16 -h-light/8-h-dark photoperiod. The 2-week-old seedlings were potted in soil and placed in a growth chamber at $22{ }^{\circ} \mathrm{C}$ and $70 \% \mathrm{RH}$ under a 16 -h-light/8-h-dark photoperiod. For stress treatments, the MS medium was supplemented with $\mathrm{TM}, \mathrm{NaCl}, \mathrm{KCl}$, paraquat, or Mannitol as necessary.

\section{Vector constructions}

For 35S:Myc-MLO-1, the $M L O$ gene fragment was first cloned into pEAZY-Blunt vector. After site-directed mutating the 484-nucleotide $\mathrm{T}$ to $\mathrm{C}$, the $M L O$ mutant fragment (named $M L O-1$ ) was cloned into the $X b a \mathrm{I} / A s c \mathrm{I}$ site of pCambia1300221-Myc vector. To produce 35S:HRD3A-GFP, the KpnI/SacI GFP fragment derived from pGFP2 plasmid was inserted into pCambia1300221-Myc, digested with $X b a \mathrm{I} / A s c \mathrm{I}$, to generate pCambia1300221-GFP. Then a 2 031-bp KpnI/KpnI fragment containing the HRD3A CDS (the Arabidopsis Information Resource locus At1g18260) was cloned into the vector pCambia1300221-GFP. The 35S:HDEL-RFP was a gift from Dr Zhen Zhu of IGDB, CAS. The CDS of HRD3B from young seedling was cloned into pEAZY-Blunt vector for sequence.

\section{Verification of the T-DNA insertion mutant}

The hrd3a-1 (SALK_10943), hrd3a-2 (SALK_050640), and $h r d 3 b-1$ (SALK_054396) seeds were obtained from the ABRC (Ohio State University, Columbus, USA). Homozygous mutants were identified by PCR from genomic DNA. For $h r d 3 a-1$ and $h r d 3 a-2$, using $H R D 3 A$ gene-specific primer (LP, 5'-TGCATTACCTTCTTCAAACACC-3'; RP, 5'-ATGCTGGTCAACCAAAGGCT-3') and T-DNA left border primers (LBb1, 5'-GCGTGGACCGCTTGCTGCAACT-3'). For $h r d 3 b-1$, using forward primer (LP3, 5'-GCCTCTGAACAGGGAAACG-3'), T-DNA left border primers (LBb1), and $H R D 3 B$ gene-specific reverse primer (RP2, 5'-TGTGCCACGTTAGCACCAA-3'). 


\section{RT-PCR amplification}

To examine the expression of HRD $3 A$ and HRD $3 B$ by RT-PCR, DNase I-treated total RNA $(2 \mu \mathrm{g})$ was denatured and subjected to reverse transcription reaction at $42{ }^{\circ} \mathrm{C}$ for $1 \mathrm{~h}$. To determine the changes of MLO-1 expression in $h r d 3 a-1$, RNA was extracted with Rneasy plant mini kit (NO.74904 QIAGEN) from $200 \mu 1$ protoplast. After being treated with DNase I, total RNA was denatured and subjected to reverse transcription reaction at $42{ }^{\circ} \mathrm{C}$ for $1 \mathrm{~h}$. For $H R D 3 A$ gene, PCR amplification was performed using $H R D 3 A$-specific forward and reverse primers (the same as LP, RP mentioned above for T-DNA verification). For $H R D 3 B$, PCR amplification was performed using $H R D 3 B$-specific primers (the same as LP3, RP2 mentioned above for T-DNA verification). Expression levels of Actin1 were monitored with forward and reverse primers (forward, 5'-CATCAGGAAGGACTTGTACGG-3'; reverse, 5'-GATGGACCTGACTCGTCATAC-3') to serve as an internal control. To find out whether $H R D 3 B$ has alternative splicing form, PCR amplification was performed using primers (LP1, 5'-CTGAGCCAGTTAGAATCCAC-3'; LP2, 5'-GGAGAGCCTATGTCAATGGAAC-3'; RP1, 5'-CATCCTTCTTGACTCCAGTG-3') near or in the putative second intron. For detecting the expression of $M O L-1$, PCR amplification was performed using $M L O$-specific primers (F, 5'-GCATTTTGTGTGGACAGTGG-3'; R, 5'-TCGTTAGGATTTAGTCTGTGC-3'). GFP was amplified with primers (F, 5'-ATGGGTAAAGGAGAAGAAC-3'; R, 5'-TTATTTGTATAGTTCATCCATG-3') as an internal control.

\section{Gene expression analysis}

The 2-week-old seedlings grown on MS plates were treated with $\mathrm{TM}, \mathrm{NaCl}$, and $\mathrm{NaCl}$ or TM plus 2-APB for corresponding times. Total RNA was isolated by $\mathrm{LiCl}$ precipitation [49]. Totally $15 \mu \mathrm{g}$ for each sample was separated on $1.2 \%(\mathrm{w} / \mathrm{v})$ agarose formaldehyde gels and transferred to Hybond-N nylon membranes (Amersham Pharmacia Biotech). Blots were probed with PCR fragments labeled with $\left(\alpha-{ }^{32} \mathrm{P}\right) \mathrm{dCTP}$ using a Ready-Primed labeling kit (Amersham International). The transcripts of ER chaperone were detected by hybridization to the following segments of the coding sequences: $B I P 1 / 2$, nucleotides 1-554; $C N X 1$, nucleotides 150-910; and PDIL1, nucleotides 444-1 291.

\section{Protein extraction and immunoblot analysis}

The 2-week-old seedlings were ground in liquid nitrogen and resuspended in $2 \times$ SDS buffer (200 mM Tris-HCl PH6.8, 4\% SDS, $0.2 \%$ bromphenol, $20 \%$ glycerol, and $0.2 \mathrm{M}$ DTT) by vortex. Total extract was boiled for $10 \mathrm{~min}$ and centrifuged at $10000 \times \mathrm{g}$ at $4{ }^{\circ} \mathrm{C}$ for $10 \mathrm{~min}$. Supernatant was subjected to protein gel blots. Antibodies and the dilutions used in these experiments were as follows: anti-Myc antibody (sc-40 Santa Cruz, 1:500), anti-GFP antibody (NO. 1814460 Roche, 1:1 000), anti-ubiquitin monoclonal antibody (raised in our laboratory) and goat anti-mouse HRPconjugated antibody (00001-1 Proteintech, 1:2 500), anti-BRI1 antibody was a gift from Zhiyong Wang of Carnegie Institution.

\section{Cell fractionation assay}

For cell fractionation analysis, HRD3A-GFP was transiently expressed in Nicotina benthamiana leaf cells. The mixture of agrobacteria containing the $35 \mathrm{~S}: H R D 3 A-G F P$ and agrobacteria containing 35S:p19 was injected into $N$. benthamiana leaves as described [30]. After incubation for $3 \mathrm{~d}$, the infiltrated parts of leaves were subjected to protein extraction followed by cell fractionation. Buffers and the procedure were as described [50, 51].

\section{Subcellular localization}

For HRD3A localization, the agrobacteria containing the 35S:HRD 3A-GFP or the mixture of agrobacteria containing the $35 \mathrm{~S}: H R D 3 A-G F P$ and agrobacteria containing $35 \mathrm{~S}: H D E L-$ $R F P$ was injected into $N$. benthamiana leaves as described [30]. Agrobacteria containing 35S:p19 was co-infiltrated to promote protein expression. Around 3 days later, GFP and RFP signals were observed and images were acquired using confocal laser scanning microscope.

\section{Protoplast transformation}

Rosette leaves of 4-6-week-old soil-grown Arabidopsis were used for Mesophyll protoplasts isolation. Protoplast transfected with plasmid based on a previously described protocol [52], except that the leaves were first sterilized in $70 \%$ ethanol for $1 \mathrm{~min}$. Transfected cells were kept in the dark at $22{ }^{\circ} \mathrm{C}$ and the protoplasts were collected after $24 \mathrm{~h}$. For detection of the Myc-MLO-1 protein level in the hrd3a-1 mutant, the 35S:Myc-MLO-1 plasmid was transformed into protoplasts of WT and hrd $3 a-1$ plants. The pGFP2 plasmid was transfected alongside for an internal control.

\section{In vivo detection of $\mathrm{H}_{2} \mathrm{O}_{2}$ in plants}

The accumulation of $\mathrm{H}_{2} \mathrm{O}_{2}$ in plant upon salt treatment was visually detected by using DAB. The 3-week-old seedlings of WT and $h r d 3 a-1$ were first treated with $300 \mathrm{mM} \mathrm{NaCl}$. Then the leaves were cut off and immerged in $1 \mathrm{mg} / \mathrm{ml}$ solution of DAB, $\mathrm{pH} 3.8$, for $8 \mathrm{~h}$ under light at $25{ }^{\circ} \mathrm{C}$. The reaction was terminated by immersion of the leaves in destaining solution (mixture of $20 \%$ acetic acid, $20 \%$ glycerol and $60 \%$ ethanol) and boiled for $10 \mathrm{~min}$. After cooling, the leaves were kept in fresh destaining solution and photographed.

\section{Chemicals and stresses treatment}

To find out whether $H R D 3 B$ gene was undergoing alternative splicing under different conditions, 2-week-old seedlings grown on MS plates were treated with different kinds of stresses and chemicals for $5 \mathrm{~h}$. The concentrations of chemicals were as follows: 5 $\mu \mathrm{g} / \mathrm{ml} \mathrm{TM}, 5 \mathrm{mM} \mathrm{AZC}, 2 \mathrm{mM}$ DTT, $150 \mathrm{mM} \mathrm{NaCl}$, 7\% glucose, $300 \mathrm{mM}$ Mannitol, $50 \mu \mathrm{M}$ ABA, $2 \mu \mathrm{M}$ IAA, $0.5 \mu \mathrm{M} \mathrm{SA}$, and 50 $\mu \mathrm{M}$ MeJA. For drought treatment, seedlings were transferred onto a filter paper in a covered Petri dish and subjected to drought treatment in an environment of $70 \%$ RH. For hot and cold treatment the temperature was $42{ }^{\circ} \mathrm{C}$ and $-2{ }^{\circ} \mathrm{C}$, respectively.

\section{Acknowledgments}

We thank Dr Jianming Li of University of Michigan for providing us with the bril-5 and bril-9 seeds, Dr Qianhua Shen from IGDB, CAS for the plasmid containing $M L O$ CDS sequence, and Dr Zhen Zhu of IGDB, CAS for 35S:HDEL-RFP plasmid. We are grateful to Dr Zhiyong Wang of Carnegie Institution for providing us with the BRI antibody and Dr Yanhai Yin from Iowa State University for providing us with the BES1 antibody. This research was supported by Grant CNSF31030047/90717006 from the National Natural Science Foundation of China and 973 Program 2011CB915402, which is a grant from National Basic Research 
Program of China.

\section{References}

1 Schubert U, Anton LC, Gibbs J, Norbury CC, Yewdell JW, Bennink JR. Rapid degradation of a large fraction of newly synthesized proteins by proteasomes. Nature 2000; 404:770774.

2 Vembar SS, Brodsky JL. One step at a time: endoplasmic reticulum-associated degradation. Nat Rev 2008; 9:944-957.

3 Lee AS. The glucose-regulated proteins: stress induction and clinical applications. Trends Biochem Sci 2001; 26:504-510.

4 Araki E, Oyadomari S, Mori M. Endoplasmic reticulum stress and diabetes mellitus. Intern Med 2003; 42:7-14.

5 Romisch K. Endoplasmic reticulum-associated degradation. Annu Rev Cell Dev Biol 2005; 21:435-456.

6 Hoseki J, Ushioda R, Nagata K. Mechanism and components of endoplasmic reticulum-associated degradation. $J$ Biochem 2010; 147:19-25.

7 Hampton RY. ER-associated degradation in protein quality control and cellular regulation. Curr Opin Cell Biol 2002; 14:476-482.

8 McBratney S, Winey M. Mutant membrane protein of the budding yeast spindle pole body is targeted to the endoplasmic reticulum degradation pathway. Genetics 2002; 162:567578.

9 Loertscher J, Larson LL, Matson CK, et al. Endoplasmic reticulum-associated degradation is required for cold adaptation and regulation of sterol biosynthesis in the yeast Saccharomyces cerevisiae. Eukaryot Cell 2006; 5:712-722.

10 Meusser B, Hirsch C, Jarosch E, Sommer T. ERAD: the long road to destruction. Nat Cell Biol 2005; 7:766-772.

11 Tanaka K, Suzuki T, Hattori N, Mizuno Y. Ubiquitin, proteasome and parkin. Biochim Biophys Acta 2004; 1695:235-247.

12 Gelman MS, Kopito RR. Rescuing protein conformation: prospects for pharmacological therapy in cystic fibrosis. $J$ Clin Invest 2002; 110:1591-1597.

13 Bays NW, Gardner RG, Seelig LP, Joazeiro CA, Hampton RY. Hrd1p/Der3p is a membrane-anchored ubiquitin ligase required for ER-associated degradation. Nat Cell Biol 2001; 3:24-29.

14 Hiraishi H, Mochizuki M, Takagi H. Enhancement of stress tolerance in Saccharomyces cerevisiae by overexpression of ubiquitin ligase Rsp5 and ubiquitin-conjugating enzymes. Biosci Biotechnol Biochem 2006; 70:2762-2765.

15 Dreher K, Callis J. Ubiquitin, hormones and biotic stress in plants. Ann Bot 2007; 99:787-822.

16 Vinocur B, Altman A. Recent advances in engineering plant tolerance to abiotic stress: achievements and limitations. Curr Opin Biotechnol 2005; 16:123-132.

17 Tuteja N. Mechanisms of high salinity tolerance in plants. Methods Enzymol 2007; 428:419-438.

18 Wang M, Xu Q, Yu J, Yuan M. The putative Arabidopsis zinc transporter ZTP29 is involved in the response to salt stress. Plant Mol Biol 2010; 73:467-479.

19 Martinez IM, Chrispeels MJ. Genomic analysis of the unfolded protein response in Arabidopsis shows its connection to important cellular processes. Plant Cell 2003; 15:561-576.

20 Kamauchi S, Nakatani H, Nakano C, Urade R. Gene expres- sion in response to endoplasmic reticulum stress in Arabidopsis thaliana. The FEBS Journal 2005; 272:3461-3476.

21 Gao H, Brandizzi F, Benning C, Larkin RM. A membranetethered transcription factor defines a branch of the heat stress response in Arabidopsis thaliana. Proc Natl Acad Sci USA 2008; 105:16398-16403.

22 Li J, Zhao-Hui C, Batoux M, et al. Specific ER quality control components required for biogenesis of the plant innate immune receptor EFR. Proc Natl Acad Sci USA 2009; 106:15973-15978.

23 Davletova S, Schlauch K, Coutu J, Mittler R. The zinc-finger protein Zat12 plays a central role in reactive oxygen and abiotic stress signaling in Arabidopsis. Plant Physiol 2005; 139:847-856.

24 Muller J, Piffanelli P, Devoto A, et al. Conserved ERAD-like quality control of a plant polytopic membrane protein. The Plant Cell 2005; 17:149-163.

25 Hong Z, Jin H, Fitchette AC, et al. Mutations of an alpha1,6 mannosyltransferase inhibit endoplasmic reticulum-associated degradation of defective brassinosteroid receptors in Arabidopsis. Plant Cell 2009; 21:3792-3802.

26 Hong Z, Jin H, Tzfira T, Li J. Multiple mechanism-mediated retention of a defective brassinosteroid receptor in the endoplasmic reticulum of Arabidopsis. Plant Cell 2008; 20:34183429.

27 Yamamoto M, Kawanabe M, Hayashi Y, Endo T, Nishikawa S. A vacuolar carboxypeptidase mutant of Arabidopsis thaliana is degraded by the ERAD pathway independently of its Nglycan. Biochem Biophys Res Commun 2010; 393:384-389.

28 Wang S, Narendra S, Fedoroff N. Heterotrimeric G protein signaling in the Arabidopsis unfolded protein response. Proc Natl Acad Sci USA 2007; 104:3817-3822.

29 Schroder M, Kaufman RJ. ER stress and the unfolded protein response. Mutat Res 2005; 569:29-63.

30 Liu L, Zhang Y, Tang S, et al. An efficient system to detect protein ubiquitination by agroinfiltration in Nicotiana benthamiana. Plant J 2010; 61:893-903.

31 Wang ZY, He JX. Brassinosteroid signal transduction--choices of signals and receptors. Trends Plant Sci 2004; 9:91-96.

32 Cheng NH, Pittman JK, Zhu JK, Hirschi KD. The protein kinase SOS 2 activates the Arabidopsis $\mathrm{H}(+) / \mathrm{Ca}(2+)$ antiporter CAX1 to integrate calcium transport and salt tolerance. $J$ Biol Chem 2004; 279:2922-2926.

33 Magnan F, Ranty B, Charpenteau M, Sotta B, Galaud JP, Aldon D. Mutations in AtCML9, a calmodulin-like protein from Arabidopsis thaliana, alter plant responses to abiotic stress and abscisic acid. Plant $J$ 2008; 56:575-589.

34 Parre E, Ghars MA, Leprince AS, et al. Calcium signaling via phospholipase $\mathrm{C}$ is essential for proline accumulation upon ionic but not nonionic hyperosmotic stresses in Arabidopsis. Plant Physiol 2007; 144:503-512.

35 Ashraf M. Biotechnological approach of improving plant salt tolerance using antioxidants as markers. Biotechnol Adv 2009; 27:84-93.

36 Apel K, Hirt H. Reactive oxygen species: metabolism, oxidative stress, and signal transduction. Annu Rev Plant Biol 2004; 55:373-399.

37 Chinta SJ, Rane A, Poksay KS, Bredesen DE, Andersen JK, Rao RV. Coupling endoplasmic reticulum stress to the cell 
death program in dopaminergic cells: effect of paraquat. Neuromolecular Med 2008; 10:333-342.

38 Yang W, Tiffany-Castiglioni E, Koh HC, Son IH. Paraquat activates the IRE1/ASK1/JNK cascade associated with apoptosis in human neuroblastoma SH-SY5Y cells. Toxicol Lett 2009; 191:203-210.

39 Torres MA, Jones JD, Dangl JL. Pathogen-induced, NADPH oxidase-derived reactive oxygen intermediates suppress spread of cell death in Arabidopsis thaliana. Nat Genet 2005; 37:1130-1134.

40 Cristovao AC, Choi DH, Baltazar G, Beal MF, Kim YS. The role of NADPH oxidase 1-derived reactive oxygen species in paraquat-mediated dopaminergic cell death. Antioxid Redox Signal 2009; 11:2105-2118.

41 Tunc-Ozdemir M, Miller G, Song L, et al. Thiamin confers enhanced tolerance to oxidative stress in Arabidopsis. Plant Physiol 2009; 151:421-432.

42 Liu JX, Srivastava R, Che P, Howell SH. An endoplasmic reticulum stress response in Arabidopsis is mediated by proteolytic processing and nuclear relocation of a membrane-associated transcription factor, bZIP28. Plant Cell 2007; 19:41114119.

43 Iwata Y, Koizumi N. An Arabidopsis transcription factor, AtbZIP60, regulates the endoplasmic reticulum stress response in a manner unique to plants. Proc Natl Acad Sci USA 2005; 102:5280-5285.

$44 \mathrm{Lu}$ DP, Christopher DA. Endoplasmic reticulum stress activates the expression of a sub-group of protein disulfide isomerase genes and AtbZIP60 modulates the response in Arabidopsis thaliana. Mol Genet Genomics 2008; 280:199210.

45 Wang D, Weaver ND, Kesarwani M, Dong X. Induction of protein secretory pathway is required for systemic acquired resistance. Science (New York, NY) 2005; 308:1036-1040.

46 Kim C, Meskauskiene R, Apel K, Laloi C. No single way to understand singlet oxygen signaling in plants. EMBO Rep 2008; 9:435-439.

47 Huang C, He W, Guo J, Chang X, Su P, Zhang L. Increased sensitivity to salt stress in an ascorbate-deficient Arabidopsis mutant. J Exp Bot 2005; 56:3041-3049.

48 Tu BP, Weissman JS. Oxidative protein folding in eukaryotes: mechanisms and consequences. J Cell Biol 2004; 164:341346.

49 Verwoerd TC, Dekker BM, Hoekema A. A small-scale procedure for the rapid isolation of plant RNAs. Nucleic Acids Res 1989; 17:2362.

50 Kim YS, Kim SG, Park JE, et al. A membrane-bound NAC transcription factor regulates cell division in Arabidopsis. Plant Cell 2006; 18:3132-3144.

51 Zhang Y, Yang C, Li Y, et al. SDIR1 is a RING finger E3 ligase that positively regulates stress-responsive abscisic acid signaling in Arabidopsis. Plant Cell 2007; 19:1912-1929.

52 Yoo SD, Cho YH, Sheen J. Arabidopsis mesophyll protoplasts: a versatile cell system for transient gene expression analysis. Nat Protoc 2007; 2:1565-1572.

(Supplementary information is linked to the online version of the paper on the Cell Research website.) 